\title{
Partial permanence and extinction on stochastic Lotka-Volterra competitive systems
}

\section{Chunwei Dong', Lei Liu' ${ }^{1 *}$ and Yonghui Sun²}

${ }^{*}$ Correspondence:
liulei_hust@hhu.edu.cn
${ }^{1}$ College of Science, Hohai
University, Nanjing, Jiangsu 210098,
China
Full list of author information is
available at the end of the article

available at the end of the article

\begin{abstract}
This paper discusses an autonomous competitive Lotka-Volterra model in random environments. The contributions of this paper are as follows. (a) Some sufficient conditions for partial permanence and extinction on this system are established; (b) By using some novel techniques, the conditions imposed on permanence and extinction of one-species are weakened. Finally, a numerical experiment is conducted to validate the theoretical findings.
\end{abstract}

Keywords: Lotka-Volterra model; random environments; Brownian motions; Itô formula; persistence in mean; extinction

\section{Introduction}

It is a usual phenomenon for two or more species who live in proximity share the same basic requirements and compete for resources, habitat, food, or territory. It is therefore very important to study the competitive models for multi-species. As we know, the well-known Lotka-Volterra model concerning ecological population modeling has received great attention and has been studied extensively owing to its theoretical and practical significance. A deterministic, competitive Lotka-Volterra system with $n$ interacting species is described by the $n$-dimensional differential equation

$$
\frac{d x_{i}(t)}{d t}=x_{i}(t)\left[b_{i}-\sum_{j=1}^{n} a_{i j} x_{j}(t)\right], \quad i=1,2, \ldots, n,
$$

where $x_{i}(t)$ represents the population size of species $i$ at time $t, b_{i}$ is the growth rate of species $i$, and $a_{i j}$ represents the effect of interspecific (if $i \neq j$ ) or intraspecific (if $i=j$ ) interaction.

On the other hand, from the biological point of view, population systems in the real world are inevitably affected by environmental noise. In practice, the growth rates are often subject to environmental noise. To obtain a more accurate description of such systems, we usually consider the stochastic perturbation of the growth rate $b_{i}$ by an average value plus an error term. Then the intrinsic growth rate depending on time becomes

$$
b_{i} \rightarrow b_{i}+\sigma_{i} \dot{B}_{i}(t)
$$

\section{空 Springer}

(c) 2015 Dong et al. This article is distributed under the terms of the Creative Commons Attribution 4.0 International License (http://creativecommons.org/licenses/by/4.0/), which permits unrestricted use, distribution, and reproduction in any medium, provided you give appropriate credit to the original author(s) and the source, provide a link to the Creative Commons license, and indicate if changes were made. 
where $\dot{B}_{i}(t)$ is a white noise. As a result, system (1.1) becomes a stochastic Lotka-Volterra competition system with $n$ interacting components as follows:

$$
d x_{i}(t)=x_{i}(t)\left[\left(b_{i}-\sum_{j=1}^{n} a_{i j} x_{j}(t)\right) d t+\sigma_{i} d B_{i}(t)\right], \quad i=1,2, \ldots, n,
$$

where $b_{i}, a_{i j}, \sigma_{i}$ are non-negative for $i, j=1,2, \ldots, n$, and $\sigma_{i}^{2}$ will be called the noise intensity matrix. Throughout this paper we always assume that the following hypothesis holds:

$$
b_{i}>0, \quad a_{i i}>0, \quad a_{i j} \geq 0 \quad(i \neq j) .
$$

It is therefore necessary to reveal how the noise affects the population systems. As a matter of fact, stochastic Lotka-Volterra competitive systems have recently been studied by many authors, for example, [1-6].

In the study of population systems, permanence and extinction are two important and interesting topics, respectively meaning that the population system will survive or die out in the future, which have received much attention (see [7-18]). Luo and Mao [15] revealed that a large white noise will force the stochastic Lotka-Volterra systems to become extinct while the population may be persistent under a relatively small white noise. Li and Mao [9] investigated a non-autonomous stochastic Lotka-Volterra competitive system, and some sufficient conditions on stochastic permanence and extinction were obtained. Li et al. [11] showed that both stochastic permanence and extinction have close relationships with the stationary probability distribution of the Markov chain. Tran and Yin [16] investigated stochastic permanence and extinction for stochastic competitive Lotka-Volterra systems using feedback controls.

However, most of the existing criteria are established for stochastic general LotkaVolterra system. Hence, one natural question arises: How to derive some criteria with less conservatism for stochastic Lotka-Volterra competitive systems? This issue constitutes the first motivation of this paper.

Moreover, most of the existing criteria are established for total permanence and total extinction. To the best of our knowledge, partial permanence and partial extinction have scarcely been investigated, which are very important properties. Is it feasible to obtain some partial permanence and extinction conditions for stochastic Lotka-Volterra competitive systems? Thus, the second purpose of this paper is to solve this interesting problem.

The rest of the paper is arranged as follows. The main results of this paper are stated in Sections 3 and 4. In Section 2, some preliminaries, definitions and lemmas are given. Sufficient conditions on persistence in mean and extinction for one-species are obtained in Section 3. Based on these sufficient conditions on one-species, sufficient criteria on partial permanence and extinction on system (1.2) are established in Section 4. Section 5 provides some numerical examples to check the effectiveness of the derived results.

\section{Notation}

Throughout this paper, unless otherwise specified, let $\left(\Omega, \mathscr{F},\left\{\mathscr{F}_{t}\right\}_{t \geq 0}, \mathbb{P}\right)$ be a complete probability space with a filtration $\left\{\mathscr{F}_{t}\right\}_{t \geq 0}$ satisfying the usual conditions (i.e., it is increasing and right continuous, while $\mathscr{F}_{0}$ contains all $\mathbb{P}$-null sets). Let $B(t)=\left(B_{t}^{1}, \ldots, B_{t}^{m}\right)$ be an $m$-dimensional Brownian motion defined on the probability space. Let $R_{+}^{n}=\left\{x \in R^{n}\right.$ : $x_{i}>0$ for all $\left.1 \leq i \leq n\right\}$. 
Lemma 2.1 ([19]) Assume that condition (1.3) holds. Then, for any given initial value $x(0) \in R_{+}^{n}$, there is a unique solution $x(t)$ to system (1.2) and the solution will remain in $R_{+}^{n}$ with probability 1 , namely

$$
P\left\{x(t) \in R_{+}^{n}, \forall t \geq 0\right\}=1 .
$$

Lemma 2.2 ([19]) Assume that condition (1.3) holds. Then, for any given initial value $x(0) \in R_{+}^{n}$, the solution $x_{i}(t)$ to system (1.2) obeys

$$
\sup _{0 \leq t<+\infty} \sum_{i=1}^{n} E x_{i}^{p}(t) \leq K_{p}, \quad i=1, \ldots, n .
$$

Definition 2.1 System (1.2) is said to be persistent in mean if there exist positive constants $\alpha_{i}, \beta_{i}$ such that the solution to system (1.2) has the following property:

$$
\begin{aligned}
& \limsup _{t \rightarrow \infty} \frac{1}{t} \int_{0}^{t} x_{i}(s) d s \leq \beta_{i} \quad \text { a.s. } i=1, \ldots, n, \\
& \liminf _{t \rightarrow \infty} \frac{1}{t} \int_{0}^{t} x_{i}(s) d s \geq \alpha_{i} \quad \text { a.s. } i=1, \ldots, n .
\end{aligned}
$$

To proceed with our study, we consider two auxiliary stochastic differential equations

$$
\begin{aligned}
& \left\{\begin{array}{l}
d y_{i}(t)=y_{i}(t)\left[\left(b_{i}-a_{i i} y_{i}(t)\right) d t+\sigma_{i} d B_{i}(t)\right], \\
y_{i}(0)=x_{i}(0), \quad i=1, \ldots, n,
\end{array}\right. \\
& \left\{\begin{array}{l}
d z_{i}(t)=z_{i}(t)\left[\left(b_{i}-\sum_{j \neq i} a_{i j} y_{j}(t)-a_{i i} z_{i}(t)\right) d t+\sigma_{i} d B_{i}(t)\right], \\
z_{i}(0)=x_{i}(0), \quad i=1, \ldots, n,
\end{array}\right.
\end{aligned}
$$

where

$$
y(t)=\left(y_{1}(t), \ldots, y_{n}(t)\right)^{T}, \quad z(t)=\left(z_{1}(t), \ldots, z_{n}(t)\right)^{T} .
$$

Lemma 2.3 Assume that condition (1.3) holds. Let $x(t)$ be a solution to system (1.2) with $x(0) \in R_{+}^{n}$, then we have

$$
z(t) \leq x(t) \leq y(t)
$$

i.e.,

$$
z_{i}(t) \leq x_{i}(t) \leq y_{i}(t), \quad i=1, \ldots, n .
$$

Proof By the Itô formula, we derive that

$$
\begin{aligned}
\frac{1}{x_{i}(t)}= & \frac{1}{x_{i}(0)} \exp \left[\left(\frac{\sigma_{i}^{2}}{2}-b_{i}\right) t+\sum_{j \neq i} a_{i j} \int_{0}^{t} x_{j}(s) d s-\sigma_{i} B_{i}(t)\right] \\
& +a_{i i} \int_{0}^{t} \exp \left[\left(\frac{\sigma_{i}^{2}}{2}-b_{i}\right)(t-s)+\sum_{j \neq i} a_{i j} \int_{s}^{t} x_{j}(\iota) d \iota\right. \\
& \left.-\sigma_{i}\left(B_{i}(t)-B_{i}(s)\right)\right] d s, \quad i=1, \ldots, n,
\end{aligned}
$$




$$
\begin{aligned}
\frac{1}{y_{i}(t)}= & \frac{1}{x_{i}(0)} \exp \left[\left(\frac{\sigma_{i}^{2}}{2}-b_{i}\right) t-\sigma_{i} B_{i}(t)\right]+a_{i i} \int_{0}^{t} \exp \left[\left(\frac{\sigma_{i}^{2}}{2}-b_{i}\right)(t-s)\right. \\
& \left.-\sigma_{i}\left(B_{i}(t)-B_{i}(s)\right)\right] d s, \quad i=1, \ldots, n,
\end{aligned}
$$

which means

$$
x_{i}(t) \leq y_{i}(t), \quad i=1, \ldots, n
$$

Applying the Itô formula to equation (2.2) yields

$$
\begin{aligned}
\frac{1}{z_{i}(t)}= & \frac{1}{x_{i}(0)} \exp \left[\left(\frac{\sigma_{i}^{2}}{2}-b_{i}\right) t+\sum_{j \neq i} a_{i j} \int_{0}^{t} y_{j}(s) d s-\sigma_{i} B_{i}(t)\right] \\
& +a_{i i} \int_{0}^{t} \exp \left[\left(\frac{\sigma_{i}^{2}}{2}-b_{i}\right)(t-s)+\sum_{j \neq i} a_{i j} \int_{s}^{t} y_{i}(\iota) d \iota\right. \\
& \left.-\sigma_{i}\left(B_{i}(t)-B_{i}(s)\right)\right] d s, \quad i=1, \ldots, n .
\end{aligned}
$$

From the representations of $y_{i}(t)$ and $z_{i}(t)$, and by (2.6) we have

$$
z_{i}(t) \leq x_{i}(t), \quad i=1, \ldots, n
$$

\section{Persistence in mean and extinction of one-species}

\subsection{Persistence of one-species}

In this section, we investigate persistence in mean and extinction of one-species for system (1.2). Now, let us present some lemmas which are essential to the proof of Theorem 3.1.

Lemma 3.1 ([20]) Let condition (1.3) hold. The solution $y_{i}(t)$ to equation (2.1) has the following property:

$$
\lim _{t \rightarrow \infty} \frac{\log y_{i}(t)}{t}=\left(b_{i}-\frac{\sigma_{i}^{2}}{2}\right) \wedge 0 \quad \text { a.s. }
$$

With the help of Lemma 3.1, we slightly improve Lemma 3.1 of [8] by weakening hypotheses posed on the coefficients of equation (2.2) as follows.

Lemma 3.2 Let condition (1.3) hold, and assume that $b_{i}-\frac{\sigma_{i}^{2}}{2}>0, b_{j}-\frac{\sigma_{j}^{2}}{2} \geq 0(i \neq j)$ and $b_{i}-\frac{\sigma_{i}^{2}}{2}-\sum_{j \neq i} \frac{a_{i j}}{a_{i j}}\left(\left(b_{j}-\frac{\sigma_{j}^{2}}{2}\right) \wedge 0\right)>0$. Then the solution to equation (2.2) has the property

$$
\lim _{t \rightarrow \infty} \frac{\log z_{i}(t)}{t}=0 \quad \text { a.s. }
$$

Theorem 3.1 Let condition (1.3) and assumptions in Lemma 3.2 hold. Then the solution to system (1.2) has the following property:

$$
\limsup _{t \rightarrow \infty} \frac{1}{t} \int_{0}^{t} x_{i}(s) d s \leq \frac{1}{a_{i i}}\left(b_{i}-\frac{\sigma_{i}^{2}}{2}\right) \text { a.s. }
$$




$$
\liminf _{t \rightarrow \infty} \frac{1}{t} \int_{0}^{t} x_{i}(s) d s \geq \frac{1}{a_{i i}}\left[\left(b_{i}-\frac{\sigma_{i}^{2}}{2}\right)-\sum_{j \neq i} \frac{a_{i j}}{a_{j j}}\left(\left(b_{j}-\frac{\sigma_{j}^{2}}{2}\right) \wedge 0\right)\right] \text { a.s., }
$$

which means the species i of system (1.2) is persistent in mean.

Proof Applying the Itô formula to equation (2.1) yields

$$
\log y_{i}(t)=\log y_{i}(0)+\left(b_{i}-\frac{\sigma_{i}^{2}}{2}\right) t-a_{i i} \int_{0}^{t} y_{i}(s) d s+\sigma_{i} B_{i}(t)
$$

Then we have

$$
\int_{0}^{t} y_{i}(s) d s=\frac{\left(b_{i}-\frac{\sigma_{i}^{2}}{2}\right)}{a_{i i}} t+\frac{\sigma_{i}}{a_{i i}} B_{i}(t)-\frac{1}{a_{i i}}\left(\log y_{i}(t)-\log y_{i}(0)\right) .
$$

Dividing both sides of (3.5) by $t$ yields

$$
\frac{1}{t} \int_{0}^{t} y_{i}(s) d s=\frac{1}{a_{i i}}\left[\frac{\log y_{i}(0)}{t}-\frac{\log y_{i}(t)}{t}+\left(b_{i}-\frac{\sigma_{i}^{2}}{2}\right)+\frac{1}{t} \int_{0}^{t} \sigma_{i} d B_{i}(s)\right] .
$$

Note that

$$
\lim _{t \rightarrow \infty} \frac{\log y_{i}(0)}{t}=0, \quad \frac{1}{t} \int_{0}^{t} \sigma_{i} d B_{i}(s)=0 \quad \text { a.s. }
$$

This implies

$$
\lim _{t \rightarrow \infty} \frac{1}{t} \int_{0}^{t} y_{i}(s) d s=-\frac{1}{a_{i i}} \lim _{t \rightarrow \infty} \frac{\log y_{i}(t)}{t}+\frac{1}{a_{i i}}\left(b_{i}-\frac{\sigma_{i}^{2}}{2}\right) .
$$

By Lemma 3.1, letting $t \rightarrow \infty$ on both sides of (3.6) yields

$$
\lim _{t \rightarrow \infty} \frac{1}{t} \int_{0}^{t} y_{j}(s) d s=\frac{1}{a_{j j}}\left(\left(b_{j}-\frac{\sigma_{j}^{2}}{2}\right) \vee 0\right) \text { a.s. }
$$

Combining Lemma 3.2 and (3.7), we can claim that

$$
\limsup _{t \rightarrow \infty} \frac{1}{t} \int_{0}^{t} x_{i}(s) d s \leq \lim _{t \rightarrow \infty} \frac{1}{t} \int_{0}^{t} y_{i}(s) d s=\frac{1}{a_{i i}}\left(b_{i}-\frac{\sigma_{i}^{2}}{2}\right) \quad \text { a.s. }
$$

Now we process to show assertion (3.3). Applying the Itô formula to $\log z_{i}(t)$ yields

$$
\begin{aligned}
\log z_{i}(t)= & \log z_{i}(0)-\int_{0}^{t}\left(\frac{\sigma_{i}^{2}}{2}-b_{i}\right) d s-a_{i i} \int_{0}^{t} z_{i}(s) d s \\
& +\sum_{j \neq i} \int_{0}^{t} a_{i j} z_{j}(s) d s+\int_{0}^{t} \sigma_{i} d B_{i}(s) .
\end{aligned}
$$


Dividing both sides of (3.9) by $t$ yields

$$
\begin{aligned}
\frac{\log z_{i}(t)}{t}= & \frac{\log z_{i}(0)}{t}-\frac{1}{t} \int_{0}^{t}\left(\frac{\sigma_{i}^{2}}{2}-b_{i}\right) d s-\frac{a_{i i}}{t} \int_{0}^{t} z_{i}(s) d s \\
& +\frac{1}{t} \sum_{j \neq i} \int_{0}^{t} a_{i j} y_{j}(s) d s+\frac{1}{t} \int_{0}^{t} \sigma_{i} d B_{i}(s) .
\end{aligned}
$$

Using Lemma 3.2 and the law of strong large number for martingale, we have

$$
\lim _{t \rightarrow \infty} \frac{1}{t} \int_{0}^{t} \sigma_{i} d B_{i}(s)=0, \quad \lim _{t \rightarrow \infty} \frac{\log z_{i}(t)}{t}=0 \quad \text { a.s. }
$$

Combining Lemma 3.2 and (3.11), letting $t \rightarrow+\infty$ on both sides of (3.9) yields

$$
\begin{aligned}
\lim _{t \rightarrow \infty} \frac{1}{t} \int_{0}^{t} z_{i}(s) d s & =\frac{1}{a_{i i}}\left[\left(b_{i}-\frac{\sigma_{i}^{2}}{2}\right)+\sum_{j \neq i} \lim _{t \rightarrow \infty} \frac{a_{i j}}{t} \int_{0}^{t} y_{j}(s) d s\right] \\
& =\frac{1}{a_{i i}}\left[\left(b_{i}-\frac{\sigma_{i}^{2}}{2}\right)-\sum_{j \neq i} \frac{a_{i j}}{a_{j j}}\left(\left(b_{j}-\frac{\sigma_{j}^{2}}{2}\right) \wedge 0\right)\right]>0 .
\end{aligned}
$$

Since $x_{i}(t) \geq z_{i}(t)$, assertion (3.3) is true. Therefore this theorem is proved.

Remark 3.1 Compared with the existing literature [8], the conditions imposed on the permanence of one-species are weaker.

Applying Lemma 3.1 to system (1.2), we have the following corollary, which coincides with Theorem 3.1 in [8].

Corollary 3.1 Let condition (1.3) hold and assume that $b_{i}-\frac{\sigma_{i}^{2}}{2}>0, b_{i}-\frac{\sigma_{i}^{2}}{2}-\sum_{j \neq i} \frac{a_{i j}}{a_{j j}}\left(b_{j}-\right.$ $\left.\frac{\sigma_{j}^{2}}{2}\right)>0$ for all $i=1, \ldots, n$. System $(1.2)$ is persistent in mean.

\subsection{Extinction of one-species}

Theorem 3.2 Let condition (1.3) hold and $x_{i}(t)$ be the solution to system (1.2) with positive initial value $x_{i}(0)$. Then we have the following assertions:

(i) If $\sigma_{i}^{2}>2 b_{i}$, the solution $x_{i}(t)$ to system (1.2) has the property that

$$
\limsup _{t \rightarrow \infty} \frac{\log x_{i}(t)}{t} \leq b_{i}-\frac{\sigma_{i}^{2}}{2} \quad \text { a.s. }
$$

That is, the species $i$ of system (1.2) will become extinct.

(ii) If $\sigma_{i}^{2}=2 b_{i}$, the solution $x_{i}(t)$ to system (1.2) has the property that

$$
\lim _{t \rightarrow \infty} x_{i}(t)=0 \quad \text { a.s. }
$$

That is, the species $i$ of system (1.2) still become extinct with probability one.

Proof The proof is rather technical, so we will divide it into two steps. The first step is to show the exponential extinction of species $i$ when $\sigma_{i}^{2}>2 b_{i}$. The second step is to show the extinction in the case of $\sigma_{i}^{2}=2 b_{i}$. 
Step 1. Applying the Itô formula to $\log x_{i}(t)$ yields

$$
\log x_{i}(t)=\log x_{i}(0)+\int_{0}^{t}\left(b_{i}-\frac{\sigma_{i}^{2}}{2}\right) d s-\sum_{j=1}^{n} a_{i j} \int_{0}^{t} x_{j}(s) d s+\int_{0}^{t} \sigma_{i} d B_{i}(s) .
$$

Dividing both sides of (3.15) by $t$ yields

$$
\begin{aligned}
\frac{\log x_{i}(t)}{t}= & \frac{\log x_{i}(0)}{t}+\frac{1}{t} \int_{0}^{t}\left(b_{i}-\frac{\sigma_{i}^{2}}{2}\right) d s \\
& -\frac{1}{t} \sum_{j=1}^{n} a_{i j} \int_{0}^{t} x_{j}(s) d s+\frac{1}{t} \int_{0}^{t} \sigma_{i} d B_{i}(s) .
\end{aligned}
$$

Using the law of strong large number for martingales, we can claim that

$$
\lim _{t \rightarrow \infty} \frac{1}{t} \int_{0}^{t} \sigma_{i} d B_{i}(s)=0 \quad \text { a.s. }
$$

Letting $t \rightarrow \infty$ yields

$$
\limsup _{t \rightarrow \infty} \frac{\log x_{i}(t)}{t} \leq b_{i}-\frac{\sigma_{i}^{2}}{2} \quad \text { a.s. }
$$

Step 2. Now, let us finally show assertion (3.14). Decompose the sample space into three mutually exclusive events as follows:

$$
\begin{aligned}
& \Omega_{i 1}=\left\{\omega: \limsup _{t \rightarrow \infty} x_{i}(t) \geq \liminf _{t \rightarrow \infty} x_{i}(t)=\gamma_{i}>0\right\} ; \\
& \Omega_{i 2}=\left\{\omega: \limsup _{t \rightarrow \infty} x_{i}(t)>\liminf _{t \rightarrow \infty} x_{i}(t)=0\right\} ; \\
& \Omega_{i 3}=\left\{\omega: \lim _{t \rightarrow \infty} x_{i}(t)=0\right\} .
\end{aligned}
$$

When $\sigma_{i}^{2}=2 b_{i}$, equation (3.16) has the following form:

$$
\frac{\log x_{i}(t)}{t}=\frac{\log x_{i}(0)}{t}-\frac{1}{t} \sum_{j=1}^{n} a_{i j} \int_{0}^{t} x_{i}(s) d s+\frac{1}{t} \int_{0}^{t} \sigma_{i} d B_{i}(s)
$$

Furthermore, we decompose the sample space into the following two mutually exclusive events according to the convergence of $\int_{0}^{\infty} x_{i}(s) d s$ :

$$
E_{i 1}=\left\{\omega: \int_{0}^{\infty} x_{i}(s) d s<\infty\right\}, \quad E_{i 2}=\left\{\omega: \int_{0}^{\infty} x_{i}(s) d s=\infty\right\} .
$$

The proof of $\lim _{t \rightarrow \infty} x_{i}(t)=0$ a.s. is equivalent to showing $E_{i 1} \subset \Omega_{i 3}, E_{i 2} \subset \Omega_{i 3}$ a.s. The strategy of the proof is as follows.

$\star$ First, by using the techniques proposed in [21], we show that $E_{i 1} \subset \Omega_{i 3}$. It is sufficient to show $P\left(E_{i 1} \cap \Omega_{i 1}\right)=0$ and $P\left(E_{i 1} \cap \Omega_{i 2}\right)=0$.

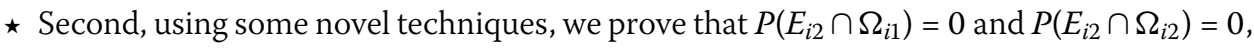
which means $E_{i 2} \subset \Omega_{i 3}$ a.s. 
Now we realize this strategy as follows.

Case 1. Let us now show $E_{i 1} \subset \Omega_{i 3}$. Clearly, $x_{i}(t) \in C\left(R_{+}, R\right)$ a.s. It is straightforward to see from $E_{i 1}$ that $\liminf _{t \rightarrow \infty} x_{i}(t)=0$ a.s. Therefore, we have obtained that $P\left(E_{i 1} \cap \Omega_{i 1}\right)=0$. Now we only need to prove that $P\left(E_{i 1} \cap \Omega_{i 2}\right)=0$. We prove it by contradiction.

If $P\left(E_{i 1} \cap \Omega_{i 2}\right)>0$, there exists a number $\epsilon>0$ such that

$$
P\left(J_{1} \cap E_{i 1}\right) \geq 2 \epsilon,
$$

where $J_{1}=\left\{\lim \sup _{t \rightarrow \infty} x_{i}(t)>2 \epsilon\right\}$. Let us now define a sequence of stopping times

$$
\begin{aligned}
& \tau_{1}=\inf \left\{t \geq 0: x_{i}(t) \geq 2 \epsilon\right\}, \quad \tau_{2 k}=\inf \left\{t \geq \tau_{2 k-1}: x_{i}(t) \leq \epsilon\right\} \\
& \tau_{2 k+1}=\inf \left\{t \geq \tau_{2 k}: x_{i}(t) \geq 2 \epsilon\right\}, \quad k=1,2, \ldots
\end{aligned}
$$

From $E_{i 1}$, we also have $E\left(I_{E_{i 1}} \int_{0}^{\infty} x_{i}(s) d s\right)<\infty$, then we compute it

$$
\begin{aligned}
E\left(I_{E_{i 1}} \int_{0}^{\infty} x_{i}(s) d s\right) & \geq \sum_{k=1}^{\infty} E\left[I_{\left\{\tau_{2 k-1}<\infty, \tau_{2 k}<\infty\right\} \cap E_{i 1}} \int_{\tau_{2 k-1}}^{\tau_{2 k}} x_{i}(s) d s\right] \\
& \geq \epsilon \sum_{k=1}^{\infty} E\left[I_{\left\{\tau_{2 k-1}<\infty\right\} \cap E_{i 1}}\left(\tau_{2 k}-\tau_{2 k-1}\right)\right],
\end{aligned}
$$

where $I_{A}$ is the indicator function for all sets $A$. Since $\tau_{2 k}<\infty$ whenever $\tau_{2 k-1}<\infty$, by the above formula, so we have

$$
\epsilon \sum_{k=1}^{\infty} E\left[I_{\left\{\tau_{2 k-1}<\infty\right\} \cap E_{i 1}}\left(\tau_{2 k-1}-\tau_{2 k}\right)\right]<\infty .
$$

On the other hand, integrating equation (1.2) from 0 to $t$ yields

$$
x_{i}(t)=x_{i}(0)+\int_{0}^{t} x_{i}(s)\left(b_{i}-\sum_{j=1}^{n} a_{i j} x_{j}(s)\right) d s+\int_{0}^{t} \sigma_{i} x_{i}(s) d B_{i}(s) .
$$

A simple computation shows that

$$
\begin{aligned}
& E\left[x_{i}^{2}(s) \cdot\left(b_{i}-\sum_{j=1}^{n} a_{i j} x_{j}(s)\right)^{2}\right] \\
& \quad \leq \frac{1}{2} E\left(x_{i}^{4}(s)\right)+\frac{1}{2} E\left[\left(b_{i}-\sum_{j=1}^{n} a_{i j} x_{j}(s)\right)^{4}\right] \\
& \quad \leq \frac{1}{2} E\left(x_{i}^{4}(s)\right)+\frac{1}{2} E\left(b_{i}^{4}+6 b_{i}^{2} \sum_{j=1}^{n} a_{i j}^{2} x_{j}^{2}(s)+\sum_{j=1}^{n} a_{i j}^{4} x_{j}^{4}(s)\right) \\
& \quad \leq \frac{1}{2} K_{4}+\frac{1}{2}\left(b_{i}^{4}+6 b_{i}^{2} \sum_{j=1}^{n} a_{i j}^{2} K_{2}+\sum_{j=1}^{n} a_{i j}^{4} K_{4}\right) \\
& =: M_{i}^{2},
\end{aligned}
$$


and

$$
E\left(\sigma_{i}^{2} \cdot x_{i}^{2}(s)\right)=\sigma_{i}^{2} \cdot E\left(x_{i}^{2}(s)\right) \leq \sigma_{i}^{2} \cdot K_{2}=: N_{i}^{2},
$$

where $K_{2}$ and $K_{4}$ are defined in Lemma 2.2. Using the Hölder inequality and BurkholderDavis-Gundy inequality (see [19]), we compute

$$
\begin{aligned}
& E\left[I_{\left.\left\{\tau_{2 k-1}<\infty\right\} \cap E_{i 1} \sup _{0 \leq t \leq T}\left|x_{i}\left(\tau_{2 k-1}+t\right)-x_{i}\left(\tau_{2 k-1}\right)\right|^{2}\right]}\right. \\
& \leq 2 E\left[I_{\left\{\tau_{2 k-1<}<\infty\right\} \cap E_{i 1}} \sup _{0 \leq t \leq T}\left|\int_{\tau_{2 k-1}}^{\tau_{2 k-1}+t}\left(x_{i}(s) \cdot\left(b_{i}-\sum_{j=1}^{n} a_{i j} x_{j}(s)\right)\right) d s\right|^{2}\right] \\
& \quad+2 E\left[I_{\left\{\tau_{2 k-1}<\infty\right\} \cap E_{i 1}} \sup _{0 \leq t \leq T}\left|\int_{\tau_{2 k-1}}^{\tau_{2 k-1}+t}\left(\sigma_{i} \cdot x_{i}(s)\right) d B_{i}(s)\right|^{2}\right] \\
& \leq 2 T E\left[I_{\left\{\tau_{2 k-1}<\infty\right\} \cap E_{i 1}} \int_{\tau_{2 k-1}}^{\tau_{2 k-1}+T}\left(x_{i}^{2}(s) \cdot\left(b_{i}-\sum_{j=1}^{n} a_{i j} x_{j}(s)\right)^{2}\right)(s) d s\right] \\
& \quad+8 E\left[I_{\left\{\tau_{2 k-1}<\infty\right\} \cap E_{i 1}} \int_{\tau_{2 k-1}}^{\tau_{2 k-1}+T}\left(\sigma_{i}^{2} \cdot x_{i}^{2}(s)\right) d s\right] \\
& \leq 2 T\left(M_{i}^{2}+4 N_{i}^{2}\right) .
\end{aligned}
$$

Furthermore, we choose $T=T(\epsilon)>0$ sufficiently small for

$$
2 T\left(M_{i}^{2}+4 N_{i}^{2}\right) \leq \epsilon^{3}
$$

It then follows from (3.22) that

$$
P\left(\left\{\tau_{2 k-1}<\infty\right\} \cap\left\{H_{k} \cap E_{i 1}\right\}\right) \leq \frac{2(T+4) T\left(M_{i}^{2}+N_{i}^{2}\right)}{\epsilon^{2}} \leq \epsilon,
$$

where

$$
H_{k}=\left\{\sup _{1 \leq t \leq T}\left|x_{i}\left(\tau_{2 k-1}+t\right)-x_{i}\left(\tau_{2 k-1}\right)\right| \geq \epsilon\right\}, \quad k=1,2, \ldots, n .
$$

Recalling the fact that $\tau_{k}<\infty$, for $k=1,2, \ldots$, whenever $\omega \in J_{1}$, we further compute

$$
\begin{aligned}
& P\left(\left\{\tau_{2 k-1}<\infty\right\} \cap\left\{H_{k}^{c} \cap E_{i 1}\right\}\right) \\
& \quad=P\left(\left\{\tau_{2 k-1}<\infty\right\} \cap E_{i 1}\right)-P\left(\left\{\tau_{2 k-1}<\infty\right\} \cap\left\{H_{k} \cap E_{i 1}\right\}\right) \\
& \quad \geq 2 \epsilon-\epsilon=\epsilon .
\end{aligned}
$$

If $\omega \in\left\{\tau_{2 k-1}<\infty\right\} \cap\left\{H_{k}^{c} \cap E_{i 1}\right\}$, note that

$$
\tau_{2 k}(\omega)-\tau_{2 k-1}(\omega) \geq T .
$$


We derive from (3.20) and (3.24) that

$$
\begin{aligned}
\infty & >\epsilon \sum_{k=1}^{\infty} E\left[I_{\left\{\tau_{2 k-1}<\infty\right\} \cap E_{i 1}}\left(\tau_{2 k}-\tau_{2 k-1}\right)\right] \\
& \geq \epsilon \sum_{k=1}^{\infty} E\left[I_{\left\{\tau_{2 k-1}<\infty\right\} \cap\left\{H_{k}^{c} \cap E_{i 1}\right\}}\left(\tau_{2 k-1}-\tau_{2 k}\right)\right] \\
& \geq \epsilon T \sum_{k=1}^{\infty} P\left(\left\{\tau_{2 k-1}<\infty\right\} \cap\left\{H_{k}^{c} \cap E_{i 1}\right\}\right) \\
& \geq \epsilon T \sum_{k=1}^{\infty} \epsilon=\infty,
\end{aligned}
$$

which is a contraction. So that $P\left(E_{i 1} \cap \Omega_{i 2}\right)=0$ holds. Therefore, we obtain that $E_{i 1} \subset \Omega_{i 3}$.

Case 2. Now, we turn to prove that $E_{i 2} \subset \Omega_{i 3}$ a.s. It is sufficient to show $P\left(E_{i 2} \cap \Omega_{i 1}\right)=0$ and $P\left(E_{i 2} \cap \Omega_{i 2}\right)=0$. We prove it by contradiction.

If $P\left(E_{i 2} \cap \Omega_{i 1}\right)>0$ holds, for any $\omega \in E_{i 2} \cap \Omega_{i 1}, \epsilon_{0} \in\left(0, \frac{\gamma_{i}}{2}\right)$, there exists $T=\left(\epsilon_{0}, \omega\right)$ such that

$$
x_{i}(t)>\gamma_{i}-\epsilon_{0}>\frac{\gamma_{i}}{2} \quad \forall t>T \text { a.s. }
$$

It then follows from (3.17) that

$$
\frac{1}{t} \int_{0}^{t} x_{i}(s) d s=\frac{1}{t} \int_{0}^{T} x_{i}(s) d s+\frac{1}{t} \int_{T}^{t} x_{i}(s) d s \geq \frac{1}{t} \int_{0}^{T} x_{i}(s) d s+\frac{t-T}{t} \frac{\gamma_{i}}{2} \quad \text { a.s. }
$$

Letting $t \rightarrow \infty$, we obtain that

$$
\liminf _{t \rightarrow \infty} \frac{1}{t} \int_{0}^{t} x_{i}(s) d s>\frac{\gamma_{i}}{2}>0 \quad \text { a.s. }
$$

This implies

$$
\limsup _{t \rightarrow \infty} \frac{\log x_{i}(t)}{t} \leq-\sum_{j=1}^{n} a_{i j} \frac{\gamma_{i}}{2}<0 \quad \text { a.s. }
$$

which contradicts the definition of $E_{i 2}$ and $\Omega_{i 1}$. So $P\left(E_{i 2} \cap \Omega_{i 1}\right)=0$ must hold.

Now we process to show $P\left(E_{i 2} \cap \Omega_{i 2}\right)>0$ is false. For this purpose, we need more notations as follows:

$$
\begin{aligned}
& \Pi_{t}^{\epsilon}(i):=\left\{0 \leq s \leq t: x_{i}(s) \geq \epsilon\right\}, \quad \delta_{t}^{\epsilon}(i):=\frac{m\left(\Pi_{t}^{\epsilon}(i)\right)}{t}, \\
& \delta^{\epsilon}(i):=\liminf _{t \rightarrow \infty} \delta_{t}^{\epsilon} i, \quad \Delta^{\epsilon}(i):=\left\{\omega \in E_{i 2} \cap \Omega_{i 2}: \delta^{\epsilon}(i)>0\right\},
\end{aligned}
$$

where $m\left(\Pi_{t}^{\epsilon}(i)\right)$ indicates the length of $\Pi_{t}^{\epsilon}(i)$. It is easy to see that $\Delta^{0}(i)=E_{i 2} \cap \Omega_{i 2}$. For any $\epsilon_{1}<\epsilon_{2}$, simple computations show that

$$
\begin{aligned}
& \Pi_{t}^{\epsilon_{1}}(i) \supset \Pi_{t}^{\epsilon_{2}}(i), \quad m\left(\Pi_{t}^{\epsilon_{1}}(i)\right) \geq m\left(\Pi_{t}^{\epsilon_{2}}(i)\right), \\
& \delta_{t}^{\epsilon_{1}}(i)=\frac{m\left(\Pi_{t}^{\epsilon_{1}}\right)(i)}{t} \geq \delta_{t}^{\epsilon_{2}}(i)=\frac{m\left(\Pi_{t}^{\epsilon_{2}}\right)(i)}{t},
\end{aligned}
$$


which implies

$$
\delta^{\epsilon_{2}}(i) \leq \delta^{\epsilon_{1}}(i), \quad \Delta^{\epsilon_{2}}(i) \subset \Delta^{\epsilon_{1}}(i) \quad \forall \epsilon_{1}<\epsilon_{2} .
$$

It is easy to observe from the continuity of probability that

$$
P\left(\Delta^{\epsilon}(i)\right) \rightarrow P\left(\Delta^{0}(i)\right)=P\left(E_{2} \cap \Omega_{2}\right) \quad \text { as } \epsilon \rightarrow 0 .
$$

If $P\left(E_{i 2} \cap \Omega_{i 2}\right)>0$, there exists $\epsilon>0$ such that $P\left(D^{\epsilon}\right)>0$. For any $\omega \in \Delta^{\epsilon}(i)$, simple computations show that

$$
\frac{1}{t} \int_{0}^{t} x_{i}(s) d s=\frac{1}{t} \int_{\Pi_{t}^{\epsilon}(i)} x_{i}(s) d s+\frac{1}{t} \int_{[0, t] \backslash \Pi_{t}^{\epsilon}} x_{i}(s) d s \geq \frac{1}{t} \int_{\Pi_{t}^{\epsilon}(i)} x_{i}(s) d s \quad \text { a.s. }
$$

By letting $t \rightarrow \infty$, we have

$$
\liminf _{t \rightarrow \infty} \frac{1}{t} \int_{0}^{t} x_{i}(s) d s \geq \liminf _{t \rightarrow \infty} \frac{1}{t} \int_{\Pi_{t}^{\epsilon}} x_{i}(s) d s \geq \delta^{\epsilon}(i) \epsilon \quad \text { a.s. }
$$

Substituting (3.26) into (3.17), we obtain that

$$
\limsup _{t \rightarrow \infty} \frac{\log x_{i}(t)}{t} \leq-\sum_{j=1}^{n} a_{i j} \delta^{\epsilon}(i) \epsilon<0 \quad \text { a.s. }
$$

This contradicts the definition of $E_{i 2}$ and $\Omega_{i 2}$. It yields the desired assertion $P\left(E_{i 2} \cap \Omega_{i 2}\right)=0$ immediately. Combining the fact $E_{i 1} \subset \Omega_{i 3}, P\left(E_{i 2} \cap \Omega_{i 1}\right)=0$ and $P\left(E_{i 2} \cap \Omega_{i 2}\right)=0$, we can claim that

$$
\lim _{t \rightarrow \infty} x_{i}(t)=0 \quad \text { a.s. }
$$

The proof is completed.

Remark 3.2 In comparison with [8] and [11], we point out that species $i$ is still extinct when $\sigma_{i}^{2}=2 b_{i}$ by using some novel stochastic analysis techniques.

Corollary 3.2 Let condition (1.3) hold and $x(t)$ be a solution to system (1.2) with positive initial value $x(0)$. Assume that there exists an integer $m, 1 \leq m<n$, such that

$$
\begin{array}{ll}
\sigma_{i}^{2}>2 b_{i}, & i=1, \ldots, m, \\
\sigma_{i}^{2}=2 b_{i}, & i=m+1, \ldots, n .
\end{array}
$$

Then we have the following assertions:

(i) For all $i=1, \ldots, n$, the solution $x_{i}(t)$ to system (1.2) has the property that

$$
\lim _{t \rightarrow \infty} \frac{\log x_{i}(t)}{t}=b_{i}-\frac{\sigma_{i}^{2}}{2} \quad \text { a.s. } i=1, \ldots, m \text {. }
$$


(ii) For all $i=m+1, \ldots, n$, the solution $x_{i}(t)$ to system (1.2) has the property that

$$
\lim _{t \rightarrow \infty} \frac{\log x_{i}(t)}{t}=0 \quad \text { a.s. } i=m+1, \ldots, n
$$

Proof By virtue of Theorem 3.2, for all $\sigma_{i}^{2}>2 b_{i}, i=1, \ldots, m$, we obtain that

$$
\limsup _{t \rightarrow \infty} \frac{\log x_{i}(t)}{t} \leq b_{i}-\frac{\sigma_{i}^{2}}{2} \quad \text { a.s. } i=1, \ldots, m \text {. }
$$

This shows that for any $\epsilon_{i} \in\left(0, \frac{\sigma_{i}^{2}}{2}-b_{i}\right)$ there is a positive random variable $T\left(\epsilon_{i}\right)$ such that

$$
x_{i}(t) \leq \exp \left\{\left(b_{i}-\frac{\sigma_{i}^{2}}{2}\right) t+\epsilon_{i} t\right\} \quad \forall t>T\left(\epsilon_{i}\right), \text { a.s. } i=1, \ldots, m,
$$

which means

$$
\int_{0}^{\infty} x_{i}(s) d s<\infty \quad \text { a.s. } i=1, \ldots, m .
$$

Then letting $t \rightarrow \infty$ on both sides of (3.16) yields

$$
\lim _{t \rightarrow \infty} \frac{\log x_{i}(t)}{t}=b_{i}-\frac{\sigma_{i}^{2}}{2} \quad \text { a.s. } i=1, \ldots, m,
$$

which is the required assertion (3.29).

Now we process to show assertion (3.30). By utilizing Theorem 3.1 and conditions (3.28), we derive

$$
\lim _{t \rightarrow \infty} x_{i}(t)=0 \quad \text { a.s. } i=m+1, \ldots, n \text {. }
$$

This implies

$$
\lim _{t \rightarrow \infty} \frac{1}{t} \int_{0}^{t} x_{i}(s) d s=0 \quad \text { a.s. } i=m+1, \ldots, n \text {. }
$$

By the law of strong large numbers for martingales and (3.31), letting $t \rightarrow \infty$ on both sides of (3.17) yields

$$
\lim _{t \rightarrow \infty} \frac{\log x_{i}(t)}{t}=0 \quad \text { a.s. } i=m+1, \ldots, n .
$$

The proof is completed.

\section{Partial permanence and extinction}

Now in this section we present conditions for system (1.2) to be partially permanent and extinct. To proceed with our study, we consider the following auxiliary stochastic equation:

$$
\left\{\begin{array}{l}
d \Phi_{i}(t)=\Phi_{i}(t)\left(b_{i}-\sum_{j=1}^{m} a_{i j} \Phi_{j}(t)\right) d t+\sigma_{i} \Phi_{i}(t) d B_{i}(t) \\
\Phi_{i}(0)=x_{i}(0), \quad i=1, \ldots, m
\end{array}\right.
$$


Theorem 4.1 Let condition (1.3) hold. Assume that there exists an integer $m, 1 \leq m<n$, such that

$$
\begin{aligned}
& b_{i}>\frac{\sigma_{i}^{2}}{2}, \quad a_{i i}-\sum_{j \neq i}^{m} a_{j i}>0, \\
& b_{i}-\frac{\sigma_{i}^{2}}{2}-\sum_{k \neq i}^{m} \frac{a_{i k}}{a_{k k}}\left(b_{k}-\frac{\sigma_{k}^{2}}{2}\right)>0, \quad i=1, \ldots, m, \\
& b_{i}<\frac{\sigma_{i}^{2}}{2}, \quad i=m+1, \ldots, n .
\end{aligned}
$$

Then we have the following assertions:

(i) For all $i=1, \ldots, m$, the solution $x(t)$ to system (1.2) has the property that

$$
\begin{aligned}
& \limsup _{t \rightarrow \infty} \frac{1}{t} \int_{0}^{t} x_{i}(s) d s \leq \frac{1}{a_{i i}}\left(b_{i}-\frac{\sigma_{i}^{2}}{2}\right) \quad \text { a.s. } i=1, \ldots, m . \\
& \liminf _{t \rightarrow \infty} \frac{1}{t} \int_{0}^{t} x_{i}(s) d s \\
& \quad \geq \frac{1}{a_{i i}}\left[\left(b_{i}-\frac{\sigma_{i}^{2}}{2}\right)-\sum_{k \neq i}^{m} \frac{a_{i k}}{a_{k k}}\left(b_{k}-\frac{\sigma_{k}^{2}}{2}\right)\right] \quad \text { a.s. } i=1, \ldots, m .
\end{aligned}
$$

That is, for each $i=1, \ldots, m$, the species $i$ of system (1.2) is persistent in mean;

(ii) For all $i=m+1, \ldots, n$, the solution $x(t)$ to system (1.2) has the property that

$$
\begin{aligned}
\limsup _{t \rightarrow \infty} \frac{\log x_{i}(t)}{t} \leq & b_{i}-\frac{\sigma_{i}^{2}}{2}-\sum_{j=1}^{m} \frac{a_{i j}}{a_{j j}}\left[\left(b_{j}-\frac{\sigma_{j}^{2}}{2}\right)\right. \\
& \left.-\sum_{k \neq j}^{m} \frac{a_{j k}}{a_{k k}}\left(b_{k}-\frac{\sigma_{k}^{2}}{2}\right)\right] \quad \text { a.s. } i=m+1, \ldots, n .
\end{aligned}
$$

That is, for each $i=m+1, \ldots, n$, the species $i$ will become extinct.

Proof We will divide the proof into two steps. The first step is to show the permanence of the top $m$ species of system (1.2). The second step is to show the extinction for the bottom $n-m$ species of system (1.2).

Step 1 . Applying the Itô formula to (4.1) yields

$$
d \log \Phi_{i}(t)=\left(b_{i}-\frac{\sigma_{i}^{2}}{2}-\sum_{j=1}^{m} a_{i j} \Phi_{j}(t)\right) d t+\sigma_{i} d B_{i}(t), \quad i=1, \ldots, m .
$$

Simple computations show that

$$
\begin{aligned}
d\left(\log x_{i}(t)-\log \Phi_{i}(t)\right)= & -\sum_{j=1}^{m} a_{i j}\left(x_{j}(t)-\Phi_{j}(t)\right) d t \\
& -\sum_{l=m+1}^{n} a_{i l} x_{l}(t) d t, \quad i=1, \ldots, m .
\end{aligned}
$$


Applying the Itô formula to $V(t)=\sum_{i=1}^{m}\left|\log x_{i}(t)-\log \Phi_{i}(t)\right|$ yields

$$
\begin{aligned}
D^{+} V(t) & =\sum_{i=1}^{m} \operatorname{sgn}\left(x_{i}(t)-\Phi_{i}(t)\right) \cdot\left[d \log x_{i}(t)-d \log \Phi_{i}(t)\right] \\
& =-\sum_{i=1}^{m} \operatorname{sgn}\left(x_{i}(t)-\Phi_{i}(t)\right) \cdot\left[\sum_{j=1}^{m} a_{i j}\left(x_{j}(t)-\Phi_{j}(t)\right)+\sum_{l=m+1}^{n} a_{i l} x_{l}(t)\right] d t \\
& \leq-\sum_{i=1}^{m} a_{i i}\left|x_{i}(t)-\Phi_{i}(t)\right| d t+\sum_{i=1}^{m} \sum_{j \neq i}^{m} a_{i j}\left|x_{j}(t)-\Phi_{j}(t)\right| d t+\sum_{i=1}^{m} \sum_{l=m+1}^{n} a_{i l} x_{l}(t) d t \\
& =-\sum_{i=1}^{m} a_{i i}\left|x_{i}(t)-\Phi_{i}(t)\right| d t+\sum_{j=1}^{m} \sum_{i \neq j}^{m} a_{j i}\left|x_{i}(t)-\Phi_{i}(t)\right| d t+\sum_{i=1}^{m} \sum_{l=m+1}^{n} a_{i l} x_{l}(t) d t \\
& =-\sum_{i=1}^{m} a_{i i}\left|x_{i}(t)-\Phi_{i}(t)\right| d t+\sum_{i=1}^{m} \sum_{j \neq i}^{m} a_{j i}\left|x_{i}(t)-\Phi_{i}(t)\right| d t+\sum_{i=1}^{m} \sum_{l=m+1}^{n} a_{i l} x_{l}(t) d t \\
& \leq-\sum_{i=1}^{m}\left(a_{i i}-\sum_{j \neq i}^{m} a_{j i}\right)\left|x_{i}(t)-\Phi_{i}(t)\right| d t+\sum_{i=1}^{m} \sum_{l=m+1}^{n} a_{i l} x_{l}(t) d t .
\end{aligned}
$$

Hence we get

$$
D^{+} V(t) \leq-\mu \sum_{i=1}^{m}\left|x_{i}(t)-\Phi_{i}(t)\right| d t+\sum_{l=m+1}^{n} \theta_{l} x_{l}(t) d t, \quad i=1, \ldots, m,
$$

where $\mu=\min _{1 \leq i \leq m}\left(a_{i i}-\sum_{j \neq i}^{m} a_{j i}\right)>0, \theta_{l}=\sum_{i=1}^{m} a_{i l} \geq 0$. We therefore have

$$
\begin{aligned}
& V(t)+\mu \int_{0}^{t} \sum_{i=1}^{m}\left|x_{i}(s)-\Phi_{i}(s)\right| d s \\
& \leq V(0)+\sum_{l=m+1}^{n} \theta_{l} \int_{0}^{t} x_{l}(s) d s, \quad i=1, \ldots, m .
\end{aligned}
$$

Letting $t \rightarrow \infty$ on both sides of (4.10) yields

$$
\begin{aligned}
\int_{0}^{\infty}\left|x_{i}(s)-\Phi_{i}(s)\right| d s & \leq \int_{0}^{\infty} \sum_{i=1}^{m}\left|x_{i}(s)-\Phi_{i}(s)\right| d s \\
& \leq \frac{1}{\mu}\left[V(0)+\sum_{l=m+1}^{n} \theta_{l} \int_{0}^{\infty} x_{l}(s) d s\right]
\end{aligned}
$$

By Theorem 3.2 and condition (4.3), we have

$$
\int_{0}^{\infty} x_{l}(s) d s<+\infty \quad \text { a.s. } l=m+1, \ldots, n .
$$

Substituting (4.12) into (4.11) yields

$$
\int_{0}^{\infty}\left|x_{i}(s)-\Phi_{i}(s)\right| d s<+\infty \quad \text { a.s. } i=1, \ldots, m .
$$


By virtue of the similar techniques proposed in Step 2 of Theorem 3.2, we have

$$
\lim _{t \rightarrow \infty}\left|x_{i}(t)-\Phi_{i}(t)\right|=0 \quad \text { a.s. } i=1, \ldots, m
$$

When condition (4.2) is satisfied, by applying Corollary 3.1 to system (4.1), we have

$$
\begin{aligned}
& \limsup _{t \rightarrow \infty} \frac{1}{t} \int_{0}^{t} \Phi_{i}(s) d s \leq \frac{1}{a_{i i}}\left(b_{i}-\frac{\sigma_{i}^{2}}{2}\right) \quad \text { a.s. } i=1, \ldots, m, \\
& \liminf _{t \rightarrow \infty} \frac{1}{t} \int_{0}^{t} \Phi_{i}(s) d s \geq \frac{1}{a_{i i}}\left[\left(b_{i}-\frac{\sigma_{i}^{2}}{2}\right)-\sum_{k \neq i}^{m} \frac{a_{i k}}{a_{k k}}\left(b_{k}-\frac{\sigma_{k}^{2}}{2}\right)\right] \quad \text { a.s. } i=1, \ldots, m .
\end{aligned}
$$

A simple computation shows that

$$
\begin{aligned}
\limsup _{t \rightarrow \infty} \frac{1}{t} \int_{0}^{t} x_{i}(s) d s & \leq \limsup _{t \rightarrow \infty} \frac{1}{t} \int_{0}^{t}\left(x_{i}(s)-\Phi_{i}(s)\right) d s+\limsup _{t \rightarrow \infty} \frac{1}{t} \int_{0}^{t} \Phi_{i}(s) d s \\
& \leq \frac{1}{a_{i i}}\left(b_{i}-\frac{\sigma_{i}^{2}}{2}\right) \quad \text { a.s. } i=1, \ldots, m,
\end{aligned}
$$

$$
\begin{aligned}
& \liminf _{t \rightarrow \infty} \frac{1}{t} \int_{0}^{t} x_{i}(s) d s \\
& \geq \liminf _{t \rightarrow \infty} \frac{1}{t} \int_{0}^{t}\left(x_{i}(s)-\Phi_{i}(s)\right) d s+\liminf _{t \rightarrow \infty} \frac{1}{t} \int_{0}^{t} \Phi_{i}(s) d s \\
& \geq \frac{1}{a_{i i}}\left[\left(b_{i}-\frac{\sigma_{i}^{2}}{2}\right)-\sum_{k \neq i}^{m} \frac{a_{i k}}{a_{k k}}\left(b_{k}-\frac{\sigma_{k}^{2}}{2}\right)\right] \quad \text { a.s. } i=1, \ldots, m .
\end{aligned}
$$

Therefore, we obtain that $x_{i}(t)$ is persistent in mean, for all $i=1, \ldots, m$.

Step 2 . For all $i=m+1, \ldots, n$, applying Itô to $\log x_{i}(t)$ yields

$$
\begin{aligned}
\frac{\log x_{i}(t)}{t}= & \frac{\log x_{i}(0)}{t}+\frac{1}{t} \int_{0}^{t}\left(b_{i}-\frac{\sigma_{i}^{2}}{2}\right) d s-\sum_{j=1}^{m} \frac{a_{i j}}{t} \int_{0}^{t} x_{j}(s) d s \\
& -\sum_{l=m+1}^{n} \frac{a_{i l}}{t} \int_{0}^{t} x_{l}(s) d s+\frac{1}{t} \int_{0}^{t} \sigma_{i} d B_{i}(s), \quad i=m+1, \ldots, n .
\end{aligned}
$$

It follows from (4.15) that

$$
\begin{aligned}
& \liminf _{t \rightarrow \infty} \frac{1}{t} \sum_{j=1}^{m} a_{i j} \int_{0}^{t} x_{j}(s) d s \\
& \quad \geq \sum_{j=1}^{m} \frac{a_{i j}}{a_{j j}}\left[\left(b_{j}-\frac{\sigma_{j}^{2}}{2}\right)-\sum_{k \neq j}^{m} \frac{a_{j k}}{a_{k k}}\left(b_{k}-\frac{\sigma_{k}^{2}}{2}\right)\right] \quad \text { a.s. } j=1, \ldots, m .
\end{aligned}
$$

By letting $t \rightarrow \infty$ on both sides of (4.16) yields. We can conclude that

$$
\begin{aligned}
\limsup _{t \rightarrow \infty} \frac{\log x_{i}(t)}{t} \leq & b_{i}-\frac{\sigma_{i}^{2}}{2}-\sum_{j=1}^{m} \frac{a_{i j}}{a_{j j}}\left[\left(b_{j}-\frac{\sigma_{j}^{2}}{2}\right)\right. \\
& \left.-\sum_{k \neq j}^{m} \frac{a_{j k}}{a_{k k}}\left(b_{k}-\frac{\sigma_{k}^{2}}{2}\right)\right] \text { a.s. } i=m+1, \ldots, n .
\end{aligned}
$$


Finally, we can get $x_{i}(t)$ will become extinct for all $i=m+1, \ldots, n$. The proof is completed.

\section{Numerical simulations}

In this paper, we have discussed the persistence in mean and extinction of system (1.2). Moreover, sufficient conditions have been established in Theorems 3.1, 3.2 and 4.1. Thus, in this section, we give out the numerical experiment for the case $n=2$ as follows to support to our results.

$$
\left\{\begin{array}{l}
d x_{1}(t)=x_{1}(t)\left[\left(0.9-0.3 x_{1}(t)-1.2 x_{2}(t)\right) d t+\sigma_{1} d B_{1}(t)\right] \\
d x_{2}(t)=x_{2}(t)\left[\left(1.1-0.3 x_{1}(t)-0.4 x_{2}(t)\right) d t+\sigma_{2} d B_{2}(t)\right] .
\end{array}\right.
$$

The existence and uniqueness of the solution follows from Lemma 2.1. We consider the solution with initial data $x_{1}(0)=1.4, x_{2}(0)=2.1$. By Matlab software, we simulate the solution to system (5.1) with different values of $\sigma_{1}$ and $\sigma_{2}$.

In Figure 1, $\sigma_{1}=0.03, \sigma_{2}=\sqrt{2.2}$. By Theorems 3.1, 3.2 and 4.1, species 1 is persistent in mean and species 2 is extinct with zero exponential extinction rate.

In Figure 2, $\sigma_{1}=0.03, \sigma_{2}=0.04$. By the conditions of Corollary 3.1, all of the species are persistent in mean.

In Figure $3, \sigma_{1}=\sqrt{1.8}, \sigma_{2}=1$.7. By Corollary 3.2, species 1 is extinct with zero exponential extinction rate and species 2 is exponentially extinct.

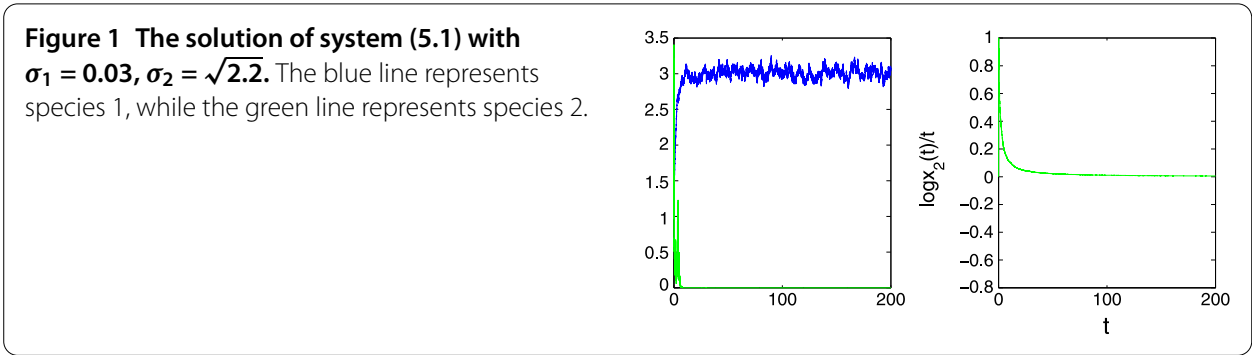

Figure 2 The solution of system (5.1) with $\sigma_{1}=\mathbf{0 . 0 3}, \sigma_{2}=\mathbf{0 . 0 4}$. The blue line represents species 1, while the green line represents species 2 .

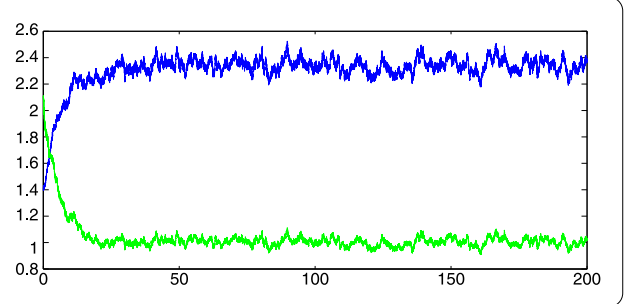

Figure 3 The solution of system (5.1) with $\sigma_{1}=\sqrt{1.8}, \sigma_{2}=1.7$. The blue line represents species 1 , while the green line represents species 2 .
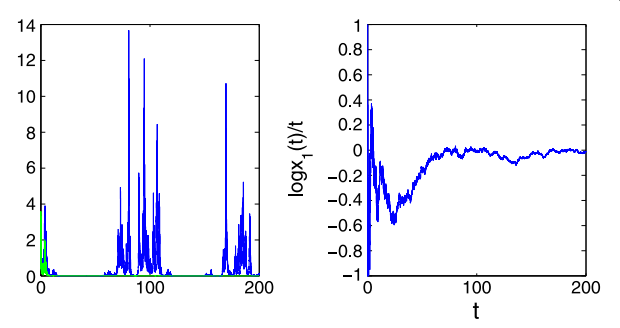


\section{Conclusions}

This paper is devoted to partial permanence and extinction on a stochastic Lotka-Volterra competitive model. Firstly, by using some novel techniques, we established some weaker sufficient conditions on the persistence in mean and extinction for one-species. Secondly, based on these sufficient conditions for one-species and some stochastic analysis techniques, sufficient criteria for ensuring the partial permanence and extinction of the populations of the $n$ different species in the ecosystem have been obtained. Finally, numerical experiment is provided to illustrate the effectiveness of our results.

\section{Competing interests}

The authors declare that they have no competing interests.

\section{Authors' contributions}

The authors have made the same contribution. All authors read and approved the final manuscript.

\section{Author details}

${ }^{1}$ College of Science, Hohai University, Nanjing, Jiangsu 210098, China. ${ }^{2}$ College of Energy and Electrical Engineering, Hohai University, Nanjing, Jiangsu 210098, China.

\section{Acknowledgements}

The authors would like to thank the editor and referees for their very important and helpful comments and suggestions. We also thank the National Natural Science Foundation of China (Grant Nos. 61304070, 11271146, 61104045, 51190102), the National Key Basic Research Program of China (973 Program) (2013CB228204).

Received: 11 February 2015 Accepted: 12 August 2015 Published online: 28 August 2015

\section{References}

1. Jiang, D, Shi, N, Li, X: Global stability and stochastic permanence of a non-autonomous logistic equation with random perturbation. J. Math. Anal. Appl. 340, 588-597 (2008)

2. Jiang, D, Shi, N: A note on non-autonomous logistic with random perturbation. J. Math. Anal. Appl. 303, 164-172 (2005)

3. Liu, M, Wang, K: Stationary distribution, ergodicity and extinction of a stochastic generalized logistic system. Appl. Math. Lett. 25, 1980-1985 (2012)

4. Liu, M, Wang, K: Persistence and extinction in stochastic nonautonomous logistic systems. J. Math. Anal. Appl. 375 , 443-457 (2011)

5. Mao, X, Marion, G, Renshaw, E: Environmental noise suppresses explosion in population dynamics. Stoch. Process. Appl. 97, 95-110 (2002)

6. Mao, X: Stationary distribution of stochastic population systems. Syst. Control Lett. 60, 398-405 (2011)

7. Bao, J, Mao, X, Yin, G, Yuan, C: Competitive Lotka-Volterra population dynamics with jumps. Nonlinear Anal. 74, 6601-6616 (2011)

8. Jiang, D, Ji, C, Li, X, O’Regan, D: Analysis of autonomous Lotka-Volterra competition systems with random perturbation. J. Math. Anal. Appl. 390, 582-595 (2012)

9. Li, X, Mao, X: Population dynamical behavior of non-autonomous Lotka-Volterra competitive system with random perturbation. Discrete Contin. Dyn. Syst. 24, 523-545 (2009)

10. Li, X, Jiang, D, Mao, X: Population dynamical behavior of Lotka-Volterra system under regime switching. Comput. Appl. Math. 232, 427-448 (2009)

11. Li, X, Gray, A, Jiang, D, Mao, X: Sufficient and necessary conditions of stochastic permanence and extinction for stochastic logistic populations under regime switching. J. Math. Anal. Appl. 376, 11-28 (2011)

12. Liu, M, Wang, K: Population dynamical behavior of Lotka-Volterra cooperative systems with random perturbations. Discrete Contin. Dyn. Syst. 33, 2495-2522 (2013)

13. Liu, M, Wang, K: Dynamics of a two-prey one-predator system in random environments. J. Nonlinear Sci. 23, 751-775 (2013)

14. Liu, M, Wang, K: Dynamics of a Leslie-Gower Holling-type II predator-prey system with Lévy jumps. Nonlinear Anal. 85, 204-213 (2013)

15. Luo, Q, Mao, X: Stochastic population dynamics under regime switching II. J. Math. Anal. Appl. 355, 577-593 (2009)

16. Tran, K, Yin, G: Stochastic competitive Lotka-Volterra ecosystems under partial observation: feedback controls for permanence and extinction. J. Franklin Inst. 351, 4039-4064 (2014)

17. Zhu, C, Yin, G: On competitive Lotka-Volterra model in random environments. J. Math. Anal. Appl. 357, 154-170 (2009)

18. Zhu, C, Yin, G: On hybrid competitive Lotka-Volterra ecosystems. Nonlinear Anal. 71, 1370-1379 (2009)

19. Mao, X: Stochastic Differential Equations and Applications. Horwood, Chichester (1997)

20. Liu, L, Shen, Y: Sufficient and necessary conditions on the existence of stationary distribution and extinction for stochastic generalized logistic system. Adv. Differ. Equ. 201510 (2015)

21. Mao, X: A note on the LaSalle-type theorems for stochastic differential delay equations. J. Math. Anal. Appl. 268, 125-142 (2002) 